\title{
Couplage dynamique - aérodynamique sur le rotor principal d'hélicoptère
}

\section{Dynamic and aerodynamical coupling on the main rotor of a helicopter}

\author{
par P. Beaumier, M. Costes \\ Département d'Aérodynamique Appliquée \\ D. Petot \\ Département de Dynamique des Structures et Systèmes Couplés \\ ONERA
}

The coupling between aerodynamic and dynamic phenomena which are necessarily found on a helicopter rotor are described. The numerical models which are being used to simulate these couplings are presented. They start from basic dynamic and aerodynamic models (beam model coupled to $2 \mathrm{D}$ quasi steady aerodynamics), used for day to day analysis, to an iterative coupling between dynamics and 3D unsteady CFD. Future evolutions of these methods are also described. They will need powerful computers to solve CFD and CSM simultaneously.

\section{I — LE PROBLÈME FLUIDE-STRUCTURE SUR L'HÉLICOPTERE}

\subsection{Le rotor articulé}

L'hélicoptère fait partie de la classe des aéronefs à voilure tournante ou giravions, pour lesquels la sustentation de l'appareil est obtenue par la rotation des pales d'un rotor qui fait office de voilure. Cela leur autorise en particulier l'accès à des conditions de vol à vitesse nulle (vol stationnaire). La spécificité de l'hélicoptère est que sa voilure tournante assure également la propulsion de l'appareil. Le rotor, appelé rotor principal, est donc l'élément essentiel de l'aérodynamique de l'appareil.

La rotation des pales entraîne une concentration des efforts vers leur extrémité, car les forces aérodynamiques sont proportionnelles au carré de la vitesse relative de la pale par rapport à l'air, induisant ainsi des moments de flexion très élevés en emplanture. Les contraintes mécaniques nécessitent l'introduction d'une articulation au niveau du moyeu qui permet d'annuler ce moment de flexion et d'autoriser ainsi à la pale de battre dans un plan qui contient la pale et son axe de rotation : c'est l'articulation de battement. En vol d'avancement, les pales rencontrent un écoulement relatif variable avec leur azimut, si bien que les phénomènes aérodynamiques sont instationnaires. On distingue deux secteurs azimutaux du disque rotor: le secteur de la pale avançante, entre les azimuts $0^{\circ}$ et $180^{\circ}$, où la pale avance par rapport au fuselage, contrairement au secteur de la pale reculante, entre $180^{\circ}$ et $360^{\circ}$. En pale avançante, la composition des vitesses de rotation et d'avancement entraîne une vitesse élevée de la pale par rapport à l'air. Un nombre de Mach incident supérieur à 0,9 peut être rencontré en extrémité de pale, source d'écoulements transsoniques. Le calage de la pale doit être faible. Au contraire, en pale reculante, les vitesses d'attaque sont faibles, le nombre de Mach incident en extrémité de pale pouvant atteindre 0,3. Il est alors nécessaire de caler la pale à une incidence élevée pour compenser la dissymétrie latérale de portance qui apparaît en raison de la différence de vitesse avec la pale avançante. Cette dissymétrie introduit un moment de roulis qui ne permet pas à l'hélicoptère de voler. De plus, elle est source de moments de flexions alternés intenses. L'articulation de battement permet de compenser naturellement cette dissymétrie. En effet, la pale étant en équilibre, l'augmentation de vitesse en pale avançante entraîne un mouvement ascendant de la pale, et son incidence aérodynamique est réduite. Au contraire, en pale reculante, la diminution de vitesse entraîne un mouvement descendant de la pale, augmentant ainsi son incidence et les efforts aérodynamiques associés.

Le mouvement de battement, indispensable pour la tenue mécanique du rotor principal et l'équilibre de l'hélicoptère, combiné à la rotation de la pale, lui fait subir une accélération fluctuante dans son plan de rotation par effet d'inertie 
(accélération de Coriolis), et donc un moment de flexion alterné autour de l'axe de rotation de la pale que l'on doit annuler par une articulation de traînée qui permet à la pale de se mouvoir librement dans son plan de rotation.

Il importe que le pilote puisse contrôler l'intensité et la direction de la force développée par le rotor. Cela est obtenu par la commande de pas, qui permet au pilote de modifier le calage des profils qui équipent la pale. On distingue $3 \mathrm{com}-$ posantes principales de la commande de pas : le pas collectif $\theta_{0}$ indépendant de l'azimut, permet d'augmenter uniformément le calage des pales sur le disque rotor et de contrôler l'intensité de la force développée par le rotor : les pas cycliques latéral $\theta_{1 \mathrm{~s}}$ et longitudinal $\theta_{1 \mathrm{c}}$ correspondent au premier harmonique de la variation de pas sur le disque rotor, et permettent de piloter la direction de la force développée par le rotor.

La cinématique des pales, composée d'un mouvement de translation, un mouvement de rotation uniforme autour de l'axe rotor, et trois mouvements de rotation dépendants du temps autour des axes de traînée, battement et pas, est complexe. Les mouvements de traînée et de battement résultent de l'équilibre instantané de la pale en réponse aux sollicitations aérodynamiques, aux efforts d'inertie, à la pesanteur et à la commande du pilote. Le pas conditionne l'équilibre global du rotor de façon à assurer une portance et une force propulsive données, et sa détermination dépend des nombreux degrés de liberté décrivant la cinématique de la pale.

\subsection{Effet de la souplesse}

Les pales sont des surfaces portantes de grand allongement, et des déformations importantes de la structure se produisent. Cela est particulièrement important en torsion, où une déformation de l'ordre du degré en pale avançante peut avoir des conséquences très importantes sur l'aérodynamique et modifier l'équilibre global et les performances du rotor. Les déformations de pale en battement sont également significatives, et elles peuvent influencer l'aérodynamique en modifiant le sillage rotor. Enfin, les sollicitations périodiques auxquelles la pale est soumise excitent les modes propres des pales, entraînant des vibrations de la structure qui sont une des grandes pénalités de l'hélicoptère.

\subsection{Moyeux souples (HMR, BMR)}

La capacité unique de l'hélicoptère à réaliser le vol stationnaire et vertical a un coût important. De très gros efforts sont réalisés afin de diminuer ces pénalités. Une simplification du moyeu du rotor principal permet d'alléger la masse des composantes mécaniques et de diminuer les coûts de maintenance. La solution apportée par les constructeurs est de remplacer les articulations mécaniques par une structure souple concentrée dans la région du moyeu. On distingue les moyeux HMR (Hingeless Main Rotor), pour lesquels la souplesse remplace les articulations de battement et de traînée, des moyeux BMR (Bearingless Main Rotor) pour lesquels la souplesse remplace les articulations de trainée, de battement, et de pas.

\subsection{Exemple : contrôle multicyclique du pas}

Les essais HART (Higher harmonic control Aeroacoustic Rotor Tests) réalisés en coopération internationale entre l'Allemagne (DLR), les Pays-Bas (DNW), les USA (NASA, US Army) et la France (ONERA) permettent d'illustrer les interactions fortes entre aérodynamique et dynamique du rotor principal [1]. Le contrôle multicyclique du pas consiste à superposer à la commande du rotor une loi de variation de pas fonction des harmoniques supérieurs de la fréquence de rotation des pales. Le but de l'application de lois de contrôle multicyclique au cours de ces essais était de modifier l'équilibre des pales de façon à minimiser les forces et moments instationnaires engendrés au niveau de la tête rotor (à l'origine des vibrations de l'hélicoptère) ou à diminuer le bruit engendré par l'interaction entre les pales et le sillage du rotor (bruit d'interaction pale-tourbillon ou BVI). Cette étude expérimentale a été réalisée sur le rotor Bol05 avec moyeu HMR dans la soufflerie du DNW. Le premier mode de torsion de la pale étant à la fréquence $3,8 \Omega(\Omega$ étant la fréquence de rotation des pales), une commande multicyclique en $3 \Omega$ permet d'obtenir une réponse significative de la pale pour une amplitude d'excitation faible $\left(0,8^{\circ}\right)$, pouvant entraîner, suivant la phase de la commande multicyclique, une diminution du bruit ou une diminution des composantes en $4 \Omega$ du torseur vibratoire à la tête rotor. La figure 1 (gauche) présente l'évolution de la composante dynamique de l'angle de torsion en extrémité de pale, ainsi qu'une comparaison avec un calcul aéro-mécanique. La présence d'une forte composante en $3 \Omega$ est bien reproduite par le calcul (bien que légèrement sous-estimée), la déflexion en torsion pouvant varier entre $-3,6^{\circ}$ et $+2,5^{\circ}$. L'influence de la torsion souple sur la portance d'une section de pale est considérable (figure 1 droite), le calcul en pale rigide donnant une variation de portance avec l'azimut très différente de celles issues du calcul souple et de l'expérience.
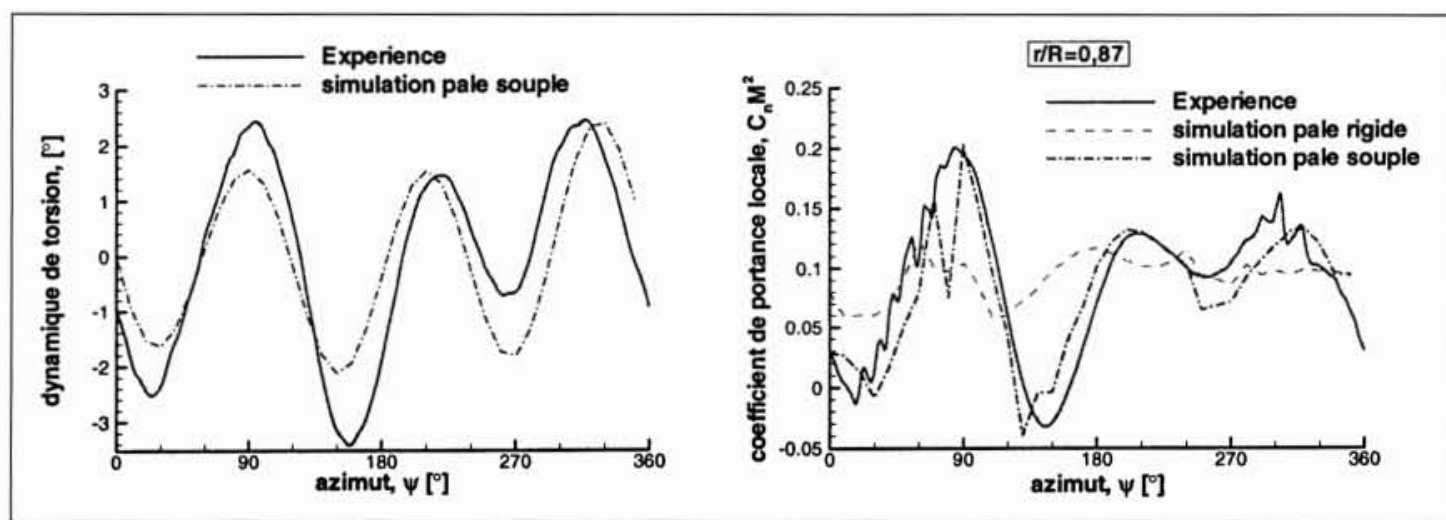
1. Influence de la sou- plesse des pales sur les déforma- tions et charges aérodyna- miques des pales.




\section{II $\square$ DESCRIPTION DES MODÈLES POUR LE ROTOR PRINCIPAL}

Sur un hélicoptère, les problèmes à résoudre sont de 3 types :

- Réponse forcée périodique,

- Intégration temporelle.

- Stabilité

Pour la réponse forcée périodique et la stabilité, les équations de l'appareil en vol avançant font apparaître des coefficients périodiques qui alourdissent grandement les calculs. Le nombre d'inconnues du problème y est multiplié par le nombre d'azimuts auquel on s'attache à obtenir la solution (20 environ). Toutes les méthodes de calcul vont donc tenter de réduire le nombre de degrés de liberté à traiter. L'intégration temporelle n'est nécessaire que pour les phases de manœuvre tandis que la réponse forcée périodique sert pour toutes les configurations de vol stabilisé. L'analyse du vol stabilisé peut toutefois aussi se faire avec une intégration temporelle si l'on dispose d'un point de départ suffisamment correct.

\subsection{Dynamique des pales}

\subsubsection{Modèles de poutres}

Les premiers calculs hélicoptères ont été effectués avec un modèle de poutre rigide, pour lequel le nombre de degrés de liberté est réduit à leur minimum : il ne reste que le battement et la traînée. Même si cette formulation pourrait encore être utilisée pour certaines applications, les calculs se font maintenant avec des modèles plus riches prenant en compte la flexibilité de la pale.

La façon la plus économe de prendre en compte la souplesse de pale est d'écrire sa déformée comme combinaison d'une série de déformées de base (on utilise les modes de pale à l'arrêt ou en rotation) [2], [3]. 6 à 8 inconnues suffisent alors en principe. Les modes sont en général obtenus par des modèles simples de type poutre comportant 20 à 30 nœuds pour discrétiser la pale. La non projection modale qui amènerait un nombre de degrés de liberté à traiter beaucoup plus important, de l'ordre de 100 par pale, est également envisageable.

\subsubsection{Méthodes CSM (Computational Structural Mechanics)}

Si l'on veut modéliser au mieux les moyeux souples modernes, et si l'on est aussi intéressé par le détail des efforts présents au sein de ces pieds de pale, une représentation fine par éléments finis devient nécessaire. L'intérêt de cette approche est de pouvoir s'appuyer sur une bibliothèque d'éléments universelle, avec toutefois l'impératif de disposer d'éléments pour lesquels la mise en rotation a été prévue, et de laisser à l'utilisateur le libre choix de la finesse de la discrétisation pour analyser un problème particulier. La réponse du rotor de l'hélicoptère s'obtient par une intégration en temps par des méthodes de type Runge-Kutta. La taille du problème devient vite prohibitive, ce qui explique le caractère exploratoire des études actuelles.

\subsection{Aérodynamique des pales}

Les outils aérodynamiques constituent une famille d'outils très vaste [4], s'étendant des modèles les plus simples présentant des temps de résolution tout à fait négligeables, aux modèles les plus lourds. Ces différents outils s'insèrent différemment dans les calculs selon qu'ils appartiennent à l'une des trois familles suivantes :

- les modèles 2D "instantanés",

- les modèles 2D "avec histoire".

- les modèles 3D.

Le sillage engendré par les pales est une partie de l'aérodynamique mais constitue souvent en lui-même un modèle à part entière qui transporte l'histoire du mouvement des pales. Cette séparation des problèmes permet une prise en compte acceptable de l'aérodynamique par des modèles simples. Lorsque la vitesse induite par le sillage n'est pas déjà incluse dans l'aérodynamique, son couplage dans le modèle fluidestructure peut se faire en parallèle de la mécanique ou de l'aérodynamique, ce qui distingue les familles suivantes : - modèles avec variables déjà périodiques (Meijer-Drees

[5], Pitt-Peters [6], voire METAR [2]),

- modèles 2D instantanés.

- modèles 3D (tourbillonnaire METAR).

A noter qu'on peut très bien rencontrer pour un même calcul une aérodynamique instantanée avec un modèle type 3D de vitesse induite (quasi-stationnaire METAR).

\subsubsection{Aérodynamique bidimensionnelle}

\section{Modèles aérodynamiques $2 D$ "instantanés"}

Les modèles aérodynamiques les plus simples sont dits bidimensionnels (2D) instantanés parce qu'ils fournissent les efforts aérodynamiques agissant sur une section en ne prenant en compte que les seules caractéristiques du mouvement instantané de cette section.

Le modèle quasi-stationnaire se contente d'associer à l'incidence et au Mach locaux des valeurs tabulées des portance, moment et traînée. Des modèles instationnaires existent également, qui prennent en compte les vitesses et accélérations du profil (Theodorsen [7]). Ces modèles sont extrêmement rapides et sont toujours utilisés pour les calculs nombreux et répétitifs en optimisation des rotors.

\section{Modèles aérodynamiques "avec histoire"}

Les modèles 2D "avec histoire" ne peuvent calculer la réponse aérodynamique qu'à partir de la connaissance de l'histoire du mouvement du profil. Il s'agit des modèles de décrochage dynamique pour lesquels on constate effectivement que la simple donnée de l'incidence ne suffit pas à déterminer si le profil est en état de décrochage ou non, cela dépendant de son état passé [8], [9], [10].

Dans une résolution pas à pas du problème instationnaire, ces modèles introduisent des degrés de liberté supplémentaires à intégrer. Pour une réponse forcée périodique, le modèle ne pourra fournir la réponse aérodynamique en une section donnée que sur tous les azimuts à la fois. Les temps de calculs sont augmentés tout en restant dans les limites du raisonnable. Ils peuvent devenir supérieurs au temps de calcul de la partie mécanique.

\subsubsection{Modélisation du sillage}

Contrairement aux aéronefs à voilure fixe, la prise en compte du sillage de façon précise est indispensable dans le calcul de l'hélicoptère. En effet, le mouvement de rotation des pales est plus rapide que la translation de l'appareil et chaque pale intercepte son propre sillage et/ou celui émis par les autres pales. L'effet du sillage est de modifier l'écoule- 

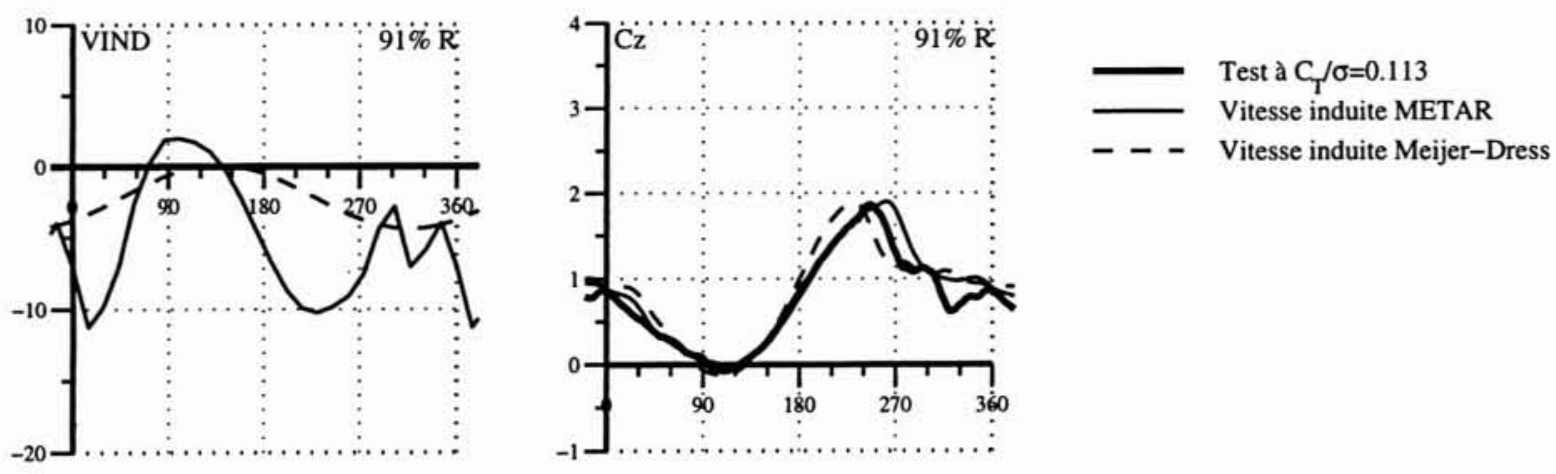

2. Comparaison des vitesses induites Meijer-Drees et METAR.

ment incident par le biais de ses vitesses induites (vitesses engendrées par le sillage au niveau du disque rotor). En pratique, on définit une incidence induite par le sillage, dont la tangente est le rapport de la vitesse induite normale au disque rotor à la vitesse de déplacement de la pale.

Les modèles de sillage les plus simples consistent à définir analytiquement les valeurs de la vitesse induite normale sans calculer le sillage proprement dit. Le modèle de MeijerDrees, utilisé dans les codes HOST, R85 [2] et ROTOR [3], décrit une évolution de la vitesse induite linéaire suivant la position radiale et harmonique suivant l'azimut. Les paramètres du modèle sont uniquement fonction de la configuration de vol.

Ces approches très simples sont acceptables pour de nombreuses applications car la contribution des sillages à l'incidence des profils est généralement faible vis-à-vis de l'influence de la cinématique de la pale. Toutefois, lorsqu'une description plus fine des phénomènes est importante, un calcul du sillage lui-même est nécessaire. Le modèle METAR développé à EUROCOPTER décrit le sillage par un ensemble de lanières tourbillonnaires d'intensité variable en envergure et en azimut de façon à vérifier le théorème de Kelvin, le sillage apparaissant ainsi comme un réseau de tourbillons quadrilatères fermés d'intensité constante reliée à la portance sur la section de pale correspondante au moment de l'émission de ces tourbillons (formule de Joukovski). La géométrie du sillage est figée et décrit un hélicoïde dont le pas est fourni par la vitesse induite de Froude. La pale ellemême est représentée par une ligne portante qui utilise les modèles 2D quasi-stationnaires décrits ci-dessus, la vitesse induite par le sillage sur la pale étant calculée par la formule de Biot et Savart. La figure 2 (gauche) montre une comparaison des vitesses induites METAR et Meijer-Drees pour le rotor 7AD, pour un fort coefficient de portance $\mathrm{C}_{\mathrm{T}} / \sigma=0,113$. En dépit de différences importantes sur les vitesses induites, l'amélioration de la portance avec le modèle METAR est assez faible (figure 2, droite), mais significative. Par contre, la richesse harmonique plus importante des vitesses induites METAR est nécessaire pour calculer le torseur vibratoire.

Lorsque la vitesse d'avancement de l'appareil devient faible, l'influence des vitesses induites devient encore plus importante, et la géométrie réelle du sillage doit être représentée. Le modèle MESIR [11], développé à l'ONERA à partir de METAR pour répondre à ce besoin, calcule itérativement la géométrie du sillage par déformations successives de façon à atteindre l'état d'équilibre pour lequel la vitesse normale au sillage s'annule. La figure 3 montre une comparaison des géométries de sillage calculées par METAR et MESIR sur le rotor Bol05 en vol de descente pour un paramètre d'avancement de 0,15 . Les déformations du sillage sous l'effet de ses propres vitesses induites sont clairement perceptibles.

\subsubsection{Méthodes CFD}

Les effets 3D compressibles instationnaires ne peuvent être décrits que grâce aux méthodes CFD (Computational Fluid Dynamics). Elles résolvent les équations de bilan de la mécanique des fluides sur un domaine de calcul discrétisé en cellules de maillage. Ce sont des méthodes de marche en temps.

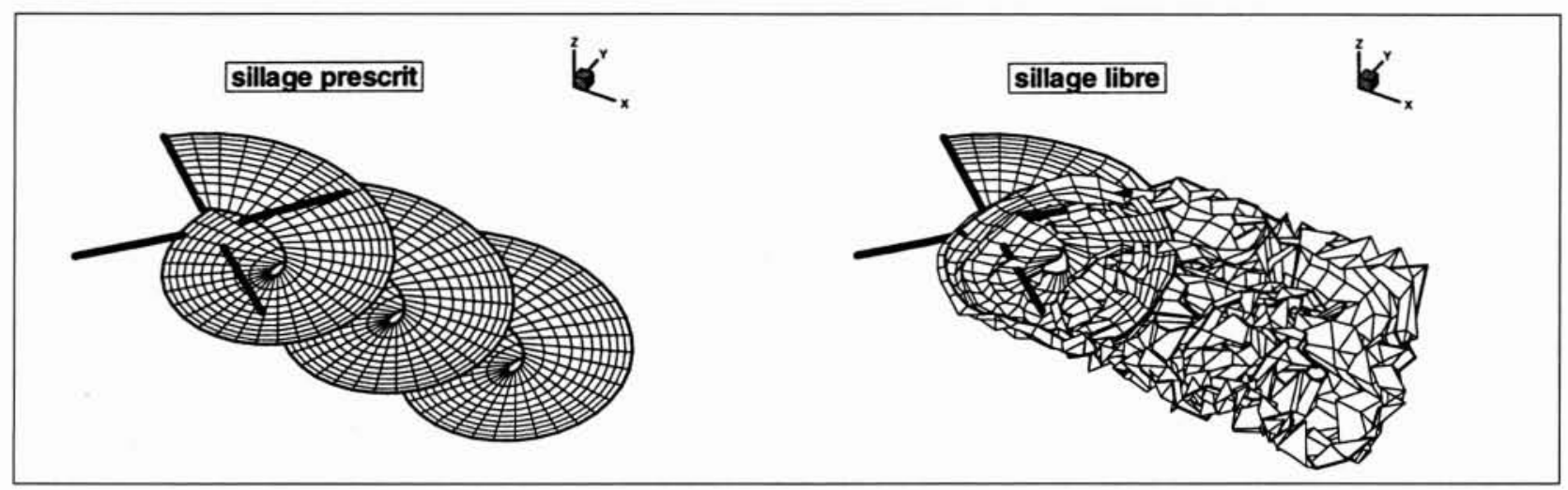

3. Comparaison des géométries de sillage prescrit et libre. 


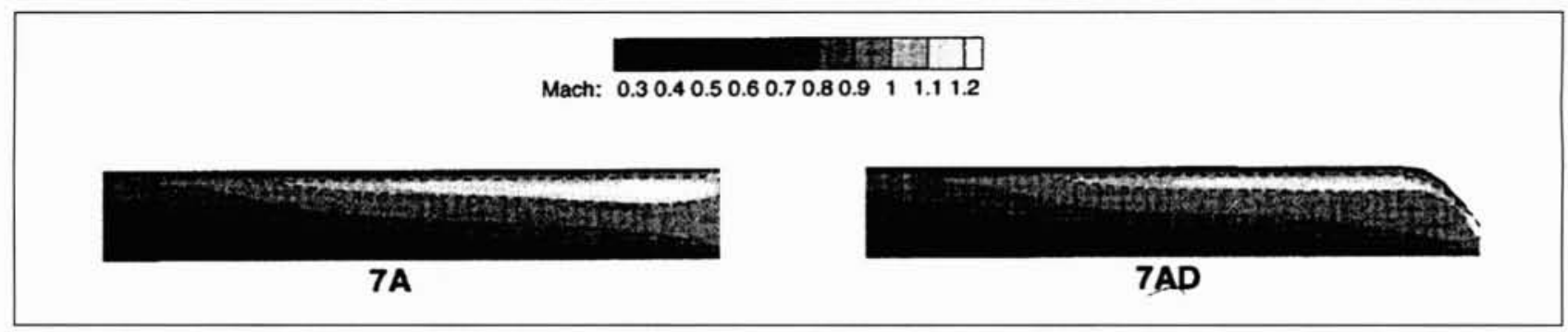

4. Lignes iso-Mach sur une pale des rotors $7 \mathrm{~A}$ et $7 \mathrm{AD}\left(\psi=120^{\circ}\right)$.

Le modèle le plus simple suppose l'écoulement non visqueux, isentropique et irrotationnel, si bien que le champ de vitesse dérive d'un potentiel. Les cinq équations décrivant le mouvement du fluide se simplifient en l'équation de continuité et l'équation de Bernoulli. Ce modèle a été appliqué dans le code FP3D à l'ONERA [12]. Le maillage de calcul structuré est lié à une pale isolée en mouvement de translation et rotation. Ce maillage peut se déformer autour de sa position nominale pour représenter les déformations de la pale. Le modèle potentiel étant irrotationnel, le calcul du rotor complet nécessite d'imposer extérieurement l'influence des autres pales et de leurs sillages ainsi que du sillage de la pale extérieur au domaine de calcul. Cela est pris en compte par l'intermédiaire des vitesses induites prescrites à la surface de la pale sous forme d'une condition de transpiration. Lorsque le maillage ne prend pas en compte les déformations, celles-ci sont également incluses dans la condition de transpiration. La dynamique de la pale doit être fournie en entrée, et la méthode fournit le champ et les efforts aérodynamiques s'exerçant sur la pale à chaque azimut du calcul.

Le principal inconvénient du modèle potentiel est son incapacité à décrire les écoulements tourbillonnaires qui se produisent dans le sillage rotor. En conservant l'hypothèse simplificatrice d'écoulement non visqueux, la forme la plus générale décrivant l'écoulement est le système des équations d'Euler. Une discrétisation par volumes finis de ces équations pour les rotors d'hélicoptères a été mise en œuvre à I'ONERA dans le code WAVES [13]. Chaque bloc de maillage comprend une pale et le domaine fluide environnant, et les blocs attachés à chacune des pales se raccordent entre eux avec nœuds coïncidants aux interfaces. Le maillage dans chacun des blocs est déformable de façon à pouvoir imposer la cinématique de la pale, et une déformation permettant de prendre en compte l'élasticité des pales peut y être superposée. Le calcul du rotor complet permet de s'affranchir du problème des vitesses induites. La dynamique des pales est une donnée d'entrée du calcul, qui fournit en retour le champ et les efforts aérodynamiques qui s'exercent sur le rotor.

On peut aller plus loin dans la généralité de la description de l'aérodynamique du rotor en résolvant les équations de Navier-Stokes moyennées. Elles consistent à rajouter aux équations d'Euler les termes de flux et les termes sources correspondant aux effets de la viscosité et de la conduction de chaleur. L'écoulement sur le rotor étant en grande partie turbulent, l'addition d'un modèle de turbulence est également nécessaire. Dans la pratique, les applications des méthodes Navier-Stokes aux rotors d'hélicoptères en vol d'avancement sont assez peu communes en raison du temps de calcul asso- cié. Leur application s'étend néanmoins et devrait rapidement permettre de fournir un champ aérodynamique visqueux utilisable pour calculer les interactions fluide-structure.

La figure 4 illustre un exemple d'information accessible aux méthodes CFD uniquement. Des lignes iso-Mach prévues par le code FP3D sur les rotors 7A et 7AD [14] sont représentées à l'azimut $120^{\circ}$ pour une configuration de vol à grande vitesse. La réduction des écoulements transsoniques apportée par l'extrémité parabolique en flèche du rotor 7AD, qui a été observée en essai à SIMA, est bien reproduite par le calcul.

\section{III - MÉTHODES DE RÉSOLUTION}

Le problème général du calcul du rotor d'hélicoptère en vol stabilisé consiste à coupler les efforts mécaniques (inertiels, contraintes, pesanteur) et les efforts aérodynamiques pour chaque azimut de la pale. Il peut faire intervenir : - les variables déjà périodiques (poutre, vitesse induite),

- les aérodynamiques 2D (quasi-stationnaire, Theodorsen, vitesse induite locale),

- les aérodynamiques 2D avec histoire (modèles de décrochage dynamique),

- les méthodes 3D (sillage tourbillonnaire, CFD, CSM).

La résolution directe des équations restitue la valeur des variables déjà périodiques. Le temps de calcul y est au minimum proportionnel au nombre de ces variables et est aussi proportionnel au temps de calcul des modèles aérodynamiques associés. L'ensemble de ces calculs peut être prohibitif en méthode directe. Pour résoudre ce problème, on utilise les stratégies suivantes :

- réduire ou supprimer complètement les variables déjà périodiques en faisant passer l'information manquante aux modèles sophistiqués par un appel interne à un ou plusieurs modèles simplifiés,

- utiliser plutôt une procédure pas à pas qui converge vers la réponse périodique pourvu que l'initialisation soit correcte.

Le savoir-faire du concepteur de la méthode de couplage permettra de choisir la combinaison de stratégies la plus efficace.

\section{- 3.1 Résolution directe des équations}

C'est la méthode utilisée par les codes de calcul hélicoptères actuels [15], qui permet la détermination des variables que l'on a appelé déjà périodiques et qui, rangées bout à bout, constituent le vecteur d'état. Le vecteur d'état permet de calculer un vecteur d'erreur qui n'est autre qu'une mesure du non-respect des équations du rotor. Ce vecteur erreur dépend des modèles aérodynamiques utilisés. Pour 


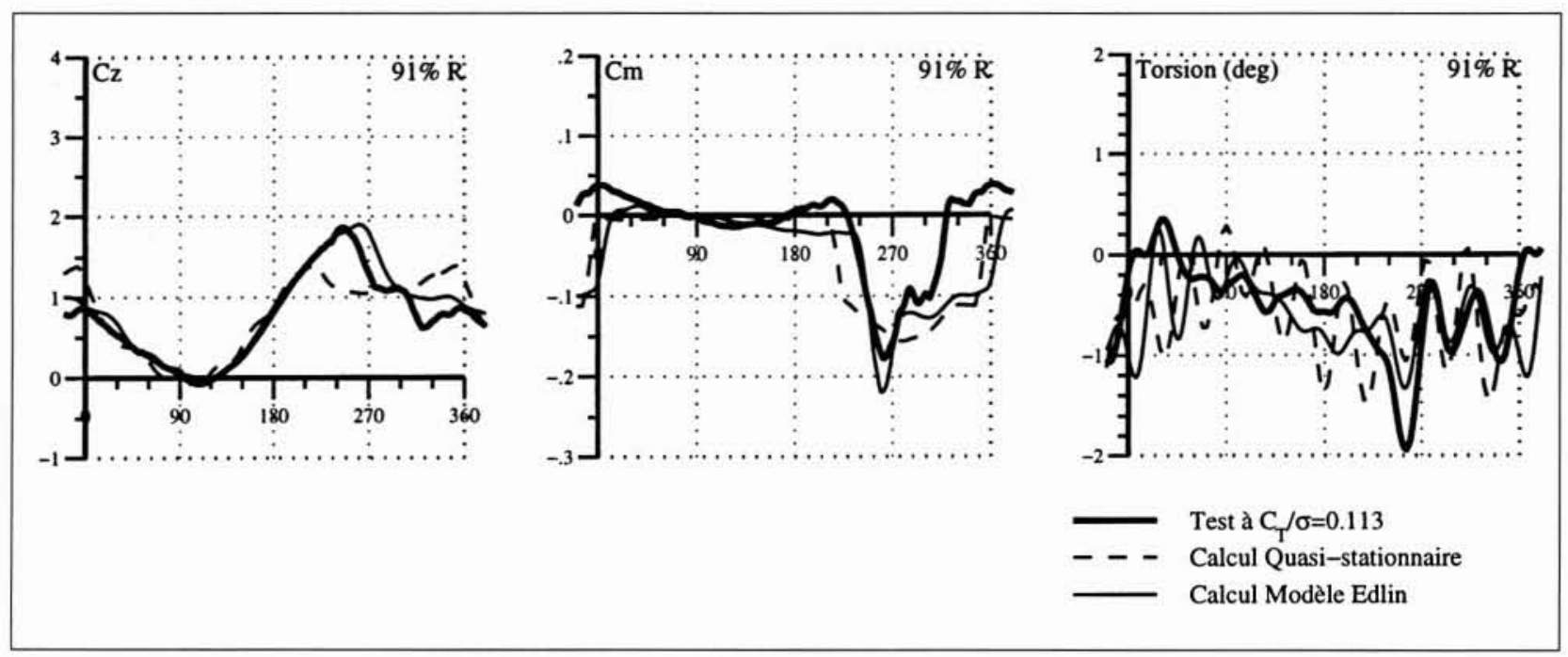

5. Influence du modèle aérodynamique.

être complet, le processus de calcul devra donc posséder 3 points d'entrée distincts pour traiter les 3 types d'aérodynamiques mentionnés : instantanées, 2D "avec histoire" et 3D.

Le système est bien sûr soluble si l'on a pu écrire autant d'équations (non linéaires) que la dimension du vecteur d'état. Un solveur mathématique recherche le vecteur d'état qui annule l'erreur et met ainsi le rotor en équilibre. Ces solveurs utilisent une méthode de Newton dans laquelle un calcul de gradient par différences finies propose une direction de recherche de la solution cherchée. Le temps de calcul global est proche de l'expression :

\section{Temps $=(5+N b d d l) *(N b$ discrétisation en azimut $) *$} (Nb Gradients) * (Temps de calcul d'un état)

dans laquelle un seul gradient suffit en général, même si le point de départ n'est pas très bon. Le temps de calcul d'un état est la somme des temps de calcul de chaque modèle mécanique ou aérodynamique.

Cette méthode de résolution nécessite que toutes les discontinuités du vecteur erreur soient soigneusement évitées, les méthodes de gradient échouant souvent dessus. Cela n'est pas toujours facile dans la zone de la pale reculante (notamment lors du passage de certaines sections de pale dans le cercle d'inversion et leur attaque par le bord de fuite). Cela constitue une limitation importante de la méthode. Son autre limitation tient dans le temps de calcul associé qui limite le nombre de degrés de liberté. Signalons toutefois qu'elle est appliquée avec succès aux 600 inconnues du modèle de vitesse induite tourbillonnaire METAR (25 rayons par 24 azimuts).

La figure 5 montre une application du code ROTOR sur le rotor $7 \mathrm{AD}$ en configuration fortement chargée. Seul le modèle instationnaire de décrochage permet de reproduire les surportances au moment du décrochage (figure 5 , gauche, $\psi \approx 270^{\circ}$ ), accompagnées d'un pic négatif du moment (figure 5, milieu). Cela permet d'améliorer l'excitation en torsion de la pale (figure 5, droite). L'aérodynamique instationnaire (Edlin) fournit bien un amortissement de torsion absent du modèle quasi-stationnaire.

\subsection{Méthodes itératives}

La résolution directe des équations couplées fluide-structure peut amener à des temps de calcul très importants (\$3.1), voire rédhibitoires si l'on souhaite utiliser des modèles aérodynamiques $3 \mathrm{D}$ (potentiel complet des vitesses, Euler, Navier-Stokes). Une alternative consiste à imaginer des méthodes itératives qui, au prix de simplifications judicieusement choisies, permettent une première approche du couplage compatible avec des temps de calculs raisonnables.

\subsubsection{Couplage sur les vitesses induites}

Le modèle de sillage prescrit décrit au $\$ 2.2 .2$ (code METAR) peut-être couplé de façon itérative à la résolution des équations de la mécanique, ce qui évite de considérer les vitesses induites comme des inconnues et réduit donc considérablement le nombre de degrés de liberté. Le principe de ce couplage est le suivant [16] :

a) initialisation de la répartition de circulation $\Gamma^{(0)}$ et de la cinématique des pales à l'aide d'un modèle de sillage analytique (Meijer-Drees par exemple : \$2.2 .2),

b) calcul de la matrice d'influence de Biot \& Savart (terme géométrique),

c) calcul des nouvelles vitesses induites $V_{i}^{(n)}$ par Biot \& Savart (et $\left.\Gamma^{(n)}\right)$,

d) recherche de l'équilibre du rotor par résolution des équations en utilisant les vitesses induites $V_{i}^{(n)}$, ce qui donne une nouvelles répartition de circulation $\Gamma^{(\mathrm{n}+1)}$.

e) rebouclage à l'étape c).

De telles itérations sont effectuées jusqu'à convergence qui s'obtient par exemple lorsque la moyenne quadratique des écarts de vitesses induites $\Delta \mathrm{V}_{\mathrm{i}}$ au niveau du disque rotor entre 2 itérations successives devient inférieure à une valeur $\varepsilon$ donnée. Le processus itératif, appliqué tel quel, est rapidement divergent parce que les vitesses induites initiales (modèle analytique) sont assez éloignées de celles obtenues dès la première itération. Un processus de sous-relaxation est nécessaire pour assurer la convergence en un nombre d'itérations généralement inférieur à 50 . 
Afin de réduire encore les temps de calcul, le couplage itératif sur les vitesses induites est effectué en résolvant lors de l'étape d) les équations de la pale rigide (dont le nombre d'inconnues est faible). Les vitesses induites obtenues à convergence sont alors directement utilisées pour la résolution des équations de la pale souple (sans couplage itératif). On suppose de cette façon que la prise en compte de la souplesse de pales ne modifie pas de façon significative les vitesses induites obtenues en pale rigide, ce qui est très souvent le cas (couplage faible). Toutefois, pour certains rotors (HMR par exemple), un couplage itératif sur les vitesses induites avec résolution des équations de la pale souple s'avère nécessaire (couplage fort).

\subsubsection{Itérations mécanique-aérodynamique}

La résolution directe des équations de la dynamique des structures et de la dynamique des fluides (même simplifiées à l'équation du potentiel pour ces dernières) est irréaliste compte tenu des moyens de calculs actuels. Afin de tenir compte d'une aérodynamique 3D instationnaire dans les calculs du rotor principal de l'hélicoptère, il est donc, là encore, indispensable de faire appel à des méthodes itératives.

Les premiers travaux de couplage réalisés à l'ONERA [17] ont consisté à associer une méthode de poutre (de type R85 ou ROTOR) à une méthode de résolution de l'équation du potentiel complet des vitesses (de type FP3D). Le couplage, schématisé par la figure 6 , se déroule de la façon suivante : a) initialisation de la solution par couplage itératif des équations de la pale souple avec un modèle de sillage (\$ 3.2.1). On obtient une répartition initiale de charges (aérodynamique 2D: $\mathrm{C}_{\mathrm{z}}{ }^{2 \mathrm{D}}, \mathrm{C}_{\mathrm{m}}{ }^{2 \mathrm{D}}, \mathrm{C}_{\mathrm{x}}{ }^{2 \mathrm{D}}$ ), une cinématique des pales et des vitesses induites initiales.

b) Résolution de l'équation du potentiel (marche en temps) sur un tour de rotor en utilisant la cinématique des pales et les vitesses induites de l'itération précédente. On obtient alors une nouvelle répartition de charges aérodynamiques : $\mathrm{C}_{\mathrm{z}}{ }^{3 \mathrm{D}}, \mathrm{C}_{\mathrm{m}}{ }^{3 \mathrm{D}}, \mathrm{C}_{\mathrm{x}}{ }^{3 \mathrm{D}}$,

c) calcul des corrections sur les coefficients de portance $\Delta \mathrm{C}_{\mathrm{z}}=\mathrm{C}_{\mathrm{z}}{ }^{3 \mathrm{D}}-\mathrm{C}_{\mathrm{z}}{ }^{2 \mathrm{D}}$, de moment $\Delta \mathrm{C}_{\mathrm{m}}=\mathrm{C}_{\mathrm{m}}{ }^{3 \mathrm{D}}-\mathrm{C}_{\mathrm{m}}{ }^{2 \mathrm{D}}$ et de traînée $\Delta \mathrm{C}_{\mathrm{x}}=\mathrm{C}_{\mathrm{x}}^{3 \mathrm{D}}-\mathrm{C}_{\mathrm{x}}^{2} 2 \mathrm{D}$,

d) nouvelle résolution des équations de la pale souple (avec couplage itératif sur le modèle de sillage) en tenant compte des corrections $\Delta \mathrm{C}_{\mathrm{z}}, \Delta \mathrm{C}_{\mathrm{m}}, \Delta \mathrm{C}_{\mathrm{x}}$,

e) rebouclage à l'étape b).

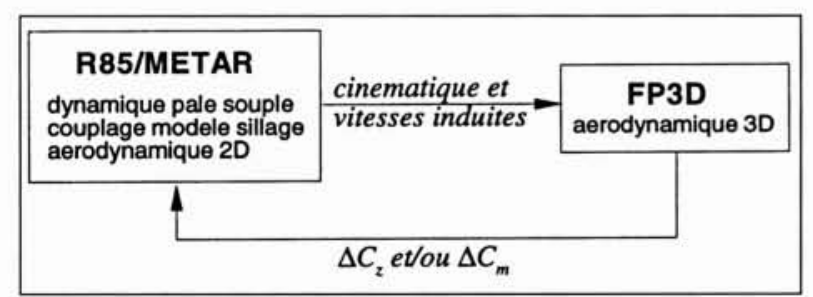

6. Schéma du couplage itératif mécanique (code R85) aérodynamique (code FP3D)

Cette méthode de couplage permet de déterminer l'équilibre du rotor en tenant compte de la souplesse des pales et des effets aérodynamiques 3D instationnaires. Toutefois, de nombreux problèmes doivent être résolus pour la mettre en œuvre. En particulier :

- la méthode "potentiel complet des vitesses" ne permet pas de calculer la traînée visqueuse des pales. Il faut donc conserver la modélisation 2D pour les coefficients de traînée et ne pas considérer la correction $\Delta \mathrm{Cx}$ dans les variables de couplage. Le problème ne se poserait pas si les équations de Navier-Stokes étaient résolues.

- la méthode "potentiel complet des vitesses" ne calcule que l'aérodynamique des sections de pale situées en envergure au-delà de $\mathrm{r} / \mathrm{R}=0,5$ (problème du cercle d'inversion). Il faut donc, lors du couplage itératif, extrapoler les corrections $\Delta \mathrm{C}_{\mathrm{z}}$ et $\Delta \mathrm{C}_{\mathrm{m}}$ entre le pied de pale et $\mathrm{r} / \mathrm{R}=0,5$. Le problème ne se poserait pas si les équations de Navier-Stokes, voire d'Euler, étaient résolues.

- une méthode de sur-relaxation sur les corrections $\Delta C_{z}$ et $\Delta C_{m}$ peut s'avérer nécessaire pour accélérer la convergence du processus itératif.

La convergence de la méthode est obtenue lorsque la répartition des efforts aérodynamiques (et des déformations de pales) entre 2 itérations successives est identique et lorsque les coefficients globaux caractéristiques de l'équilibre du rotor (pas collectif et cyclique, puissance consommée...) ne varient plus au cours des itérations.

Un exemple de convergence d'un calcul itératif (couplage R85/FP3D sur le rotor 7A) est illustré sur la figure 7. Dans ce cas, 4 itérations ont tout d'abord été effectuées en prenant comme variable de couplage uniquement les corrections $\Delta \mathrm{C}_{z}$ sur les coefficients de portance ; lors des 8 itérations suivantes, les deux variables de couplage $\Delta C_{z}$ et $\Delta C_{m}$ sont utilisées simultanément. Au bout des 12 itérations de couplage, on observe une très bonne convergence du pas collectif $\theta_{0}$ (fig. 7 , gauche), du pas cyclique $\theta_{1 \mathrm{~s}}$ (fig. 7 , milieu) et du coefficient de couple $\mathrm{C}_{\mathrm{b}}$ (fig. 7, droite). Dans le cas présenté, le couplage n'améliore pas la prévision du pas collectif mais améliore légèrement la prévision du pas cyclique $\theta_{1 \mathrm{~s}}$ et sensiblement celle du coefficient de couple $\mathrm{C}_{\mathrm{b}}$. La répartition azimutale des coefficients de portance $\mathrm{C}_{\mathrm{n}} \mathrm{M}^{2}$ vers l'extrémité de pale (fig. 8 , gauche) n'est pas améliorée : en particulier, le pic de portance négative en pale avançante (entre les azimuts $\psi=90^{\circ}$ et $\psi=140^{\circ}$ ) est toujours nettement sous-estimé par rapport à l'expérience. En revanche, le calcul avec couplage permet une amélioration très nette de la prévision des coefficients de moment $\mathrm{C}_{\mathrm{m}} \mathrm{M}^{2}$ vers l'extrémité de pale (fig. 8 , milieu), particulièrement dans la zone de la pale arrière (entre $\psi=0^{\circ}$ et $30^{\circ}$ et entre $\psi=270^{\circ}$ et $360^{\circ}$ ). Par suite, les déformations de torsion sont mieux prévues dans cette zone (fig. 8, droite). On note également que le calcul couplé permet d'enrichir le contenu harmonique de la réponse en torsion des pales, ce qui est en meilleur accord avec l'expérience que le calcul non couplé, même si l'accord calcul-expérience reste imparfait.

\subsection{Couplage consistant en temps (perspectives)}

Les méthodes de couplage actuellement utilisées permettent d'améliorer la description des efforts aérodynamiques dans le calcul d'équilibre du rotor. Cependant, ce type d'approche reste limité aux cas où l'interaction entre le fluide et la structure reste modérée, car l'influence de l'aérodynamique 3D instationnaire apparaît comme une correction de l'aérodynamique $2 \mathrm{D}$, faisant ainsi l'hypothèse implicite que ces corrections restent faibles vis-à-vis des valeurs issues du modèle simplifié. De plus, pour les phénomènes fortement instationnaires, l'interaction fluide-structure à haute fréquence peut être filtrée par ce couplage. Les algorithmes de 


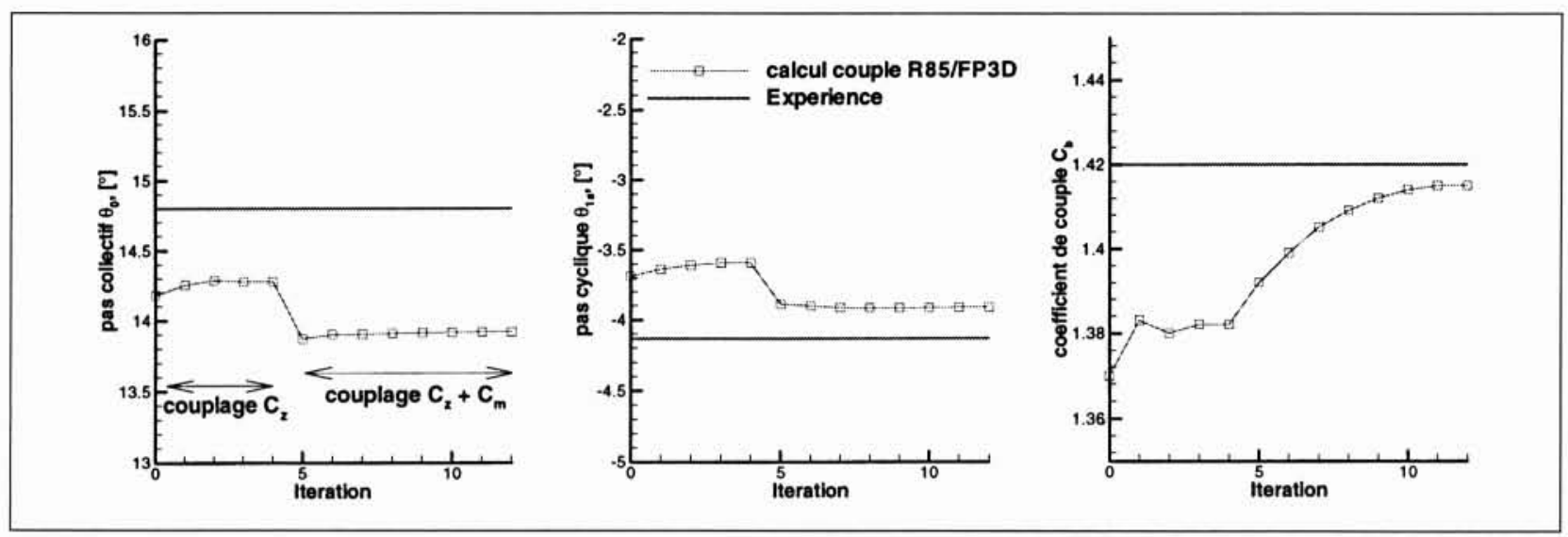

7. Convergence des grandeurs globales au cours du couplage itératif R85/FP3D.

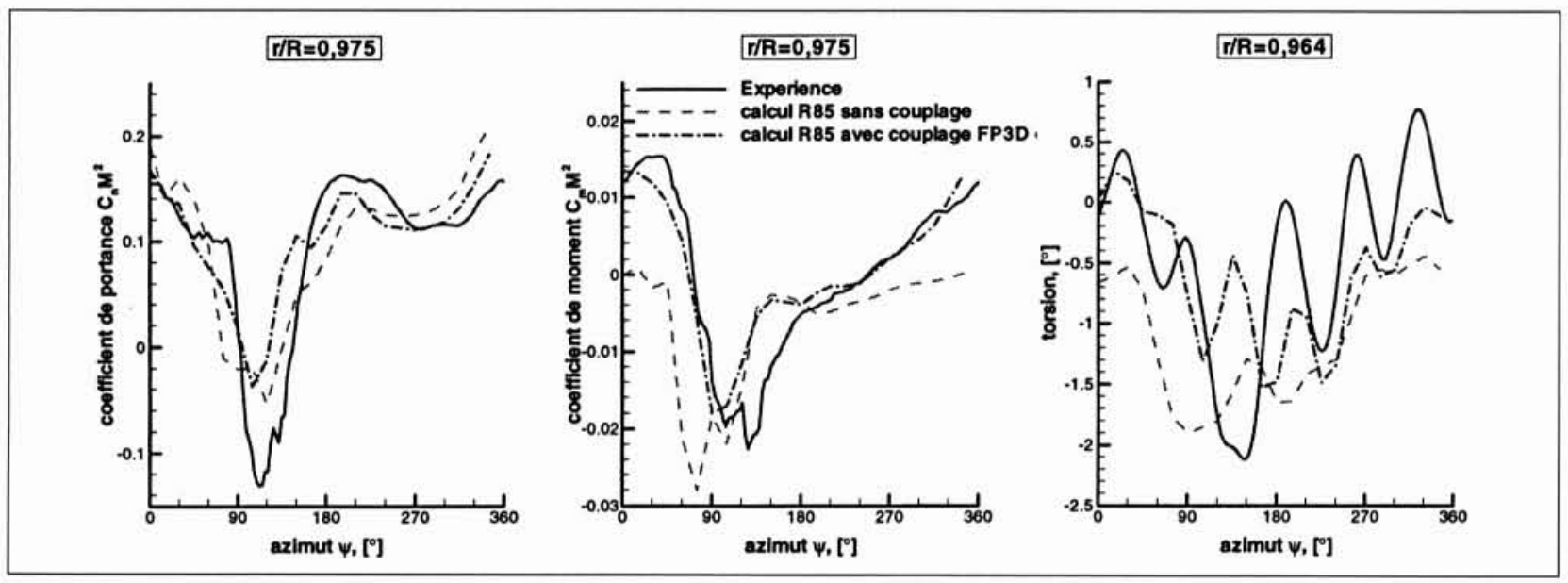

8. Influence du couplage itératif R85/FP3D sur les répartitions de charge, moment et torsion.

couplage devront être modifiés pour mieux décrire à chaque instant l'interaction mutuelle entre le fluide et la structure, l'aérodynamique 3D instationnaire venant se substituer à l'aérodynamique simplifiée des méthodes du $\$ 3.1$. Ce couplage consistant en temps est actuellement au stade de l'étude préliminaire, car les problèmes à résoudre sont nombreux. Du point de vue numérique, le temps de calcul associé au modèle aérodynamique est extrêmement important, avec des pas de temps de l'ordre du dixième de degré d'azimut et des maillages de plusieurs millions de points. Une optimisation de l'algorithme de couplage afin d'alléger le nombre d'appels au modèle aérodynamique, mais aussi une amélioration de l'efficacité de la résolution numérique du problème aérodynamique sont indispensables. De ce point de vue, la résolution du problème dynamique conjointement à la convergence du calcul aérodynamique doit être envisagée. Les techniques de calcul de gradient directement par la méthode CFD, utilisées en optimisation numérique, pourraient également s'avérer très utiles dans ce contexte. Par ailleurs, la recherche de l'équilibre entre la dynamique et l'aérodynamique peut amener les pales dans des régimes transitoires où le fonctionnement aérodynamique est peu réaliste. Les méthodes de calcul devront être extrêmement robustes pour fournir une solution et le couplage suffisamment stable pour ramener le rotor dans un domaine plus réaliste. En effet, un tel couplage présente d'autant plus d'intérêt que l'on se trouve aux limites du domaine de vol du rotor où les techniques plus simples actuellement utilisées décrivent mal la physique. De ce point de vue, les méthodes de calcul aérodynamiques doivent donc également progresser afin de mieux décrire ces phénomènes "aux limites".

De même, les modèles de poutre utilisés pour la dynamique des pales ont des limitations qui deviennent d'autant plus apparentes que le modèle aérodynamique associé progresse, mais aussi que la technologie de conception des pales évolue. L'introduction des moyeux HMR et BMR nécessite une résolution fine du moyeu afin de calculer la dynamique de la pale. Des fonctionnements non linéaires de la structure sont ainsi introduits par ces nouveaux moyeux. De plus, l'évolution de la géométrie des pales vers des formes complexes, avec effilements multiples, flèche évolutive,... introduit un couplage beaucoup plus important entre les différents modes propres de la pale, source de nouvelles non linéarités de la structure. L'introduction des méthodes CSM pour le calcul de la structure du rotor est en cours à l'ONERA. Elle permet une prise en compte naturelle de ces non linéarités et une description fine des contraintes s'exerçant dans le volume de la pale. Bien entendu, l'appel à ce nouveau type de méthodes a un coût important, et les premières applications utiliseront une aérodynamique simplifiée car la résolution par éléments finis en 3D instationnaire de la dynamique de la pale est un problème en soi. A terme, la résolution simultanée du problème fluide-structure par couplage CFD-CSM sur le rotor principal d'hélicoptère est pré- 
vue, mais il est certain qu'un progrès significatif, à la fois au niveau des techniques numériques et des capacités des ordinateurs, est indispensable afin de pouvoir traiter ce problème complexe.

\section{IV - CONCLUSIONS}

Le couplage fluide-structure est un phénomène inhérent au rotor principal d'hélicoptère. En effet, la tenue mécanique et la pilotabilité du rotor n'est rendue possible que par l'introduction d'articulations entre l'axe du rotor et les pales, cellesci se trouvant alors constamment en équilibre sous l'effet des forces d'inertie, des forces aérodynamiques et de la pesanteur. Le développement de moyens de conception des rotors a nécessité très tôt de développer des outils capables de résoudre ce couplage. Ce type de méthodes fait appel à une aérodynamique simplifiée, généralement $2 \mathrm{D}$ et quasi-stationnaire sur la base des polaires de profil qui équipent la pale. Le modèle dynamique est souvent plus sophistiqué, mais il fait appel à un modèle de poutre et utilise la base des modes propres de la pale pour décrire ses déformations. Grâce à ces simplifications, le couplage est résolu en traitant simultanément les problèmes dynamiques et aérodynamiques. Moyennant quelques corrections semi-empiriques des modèles, cette résolution permet une estimation assez correcte de la cinématique, des déformations et des performances du rotor. Des améliorations du modèle aérodynamique, comme l'introduction de modèles instationnaires ou une représentation du sillage tourbillonnaire permet d'étendre la validité de cette approche, bien que dans ce dernier cas la résolution simultanée de la dynamique et de l'aérodynamique n'est plus aussi évidente en raison du grand nombre de degrés de liberté ainsi introduits.

Néanmoins, une validation fine de ces méthodes montre que la prévision de l'aérodynamique de la pale peut être grandement améliorée en utilisant une aérodynamique 3D compressible et instationnaire. La prise en compte de ces corrections 3D instationnaires dans le calcul de la dynamique de la pale a été réalisée par couplage itératif entre les méthodes CFD et les codes de dynamique. Ce couplage, réalisé uniquement sur la portance et/ou le moment de tangage des profils, converge rapidement pour les configurations de vol couramment rencontrées, ce qui implique que les corrections 3D ne sont pas trop importantes vis-à-vis de l'équilibre obtenu avec une aérodynamique simplifiée. Cependant, le calcul de configurations de vol plus difficiles où le constructeur souhaite améliorer les performances de ses appareils nécessitera de faire appel à des méthodes de couplage plus directes entre une aérodynamique $3 \mathrm{D}$, visqueuse, compressible et instationnaire et la dynamique de la pale. A plus long terme, l'intégration de modèles $3 \mathrm{D}$ de la structure de la pale, aujourd'hui en cours de développement, dans le calcul de l'interaction dynamique-aérodynamique sera réalisée. Il nécessite néanmoins un progrès important dans l'efficacité des techniques numériques (en CFD et en CSM) et un accroissement de la puissance des calculateurs.

\section{RÉFÉRENCES}

[1] R. Kube, W.R. Splettstoesser, W. Wagner, U. Seellhorst, Y.H. Yu, C. Tung, P. Beaumier, J. Prieur, G. Rahier, P. Spiegel, A. Boutier, T.F. Brooks, C.L. Burley, D.D. Boyd JR., E. Mercker, K. Pengel, "HHC aeroacoustic rotor tests in the German Dutch wind tunnel : improving physical understanding and prediction codes", AHS 52nd Annual Forum, Washington D.C., June 4-6, 1996.

[2] G. Arnaud, F. Toulmay, B. Benort, "Améliorations du modèle aérodynamique du code rotor hélicoptère R85", 28ème Colloque d'Aérodynamique Appliquée. AAAF, ISL, 1991.

[3] J. Bessone, D. Petot, "Calculs du comportement aéroélastique des rotors comparés à l'expérience", La Recherche Aérospatiale, 1995, no I. pp. 3-14.

[4] M. Costes, P. Beaumier, N. Bettschart, P. Gardarein, "Méthodes de calcul aérodynamiques appliquées aux hélicoptères". La Recherche Aérospatiale, 1995, no 3. pp. 167-184.

[5] J.M. Drees, "A theory of airflow through rotors and its applications to some helicopter problems". Journal of the Helicopter Association of Great Britain, July 1949.

[6] D.M. PItT, D.A. Peters, "Theoretical prediction of dynamic inflow derivatives", Vertica, Vol 15, no 1, pp. 21-34, March 1981.

[7] T. THEODORSEN, "General theory of aerodynamic instability", NACA report no 496, January 1945.

[8] D. Peтot, "Modélisation du décrochage dynamique par équations différentielles", La Recherche Aérospatiale, 1989, no 5.

[9] V.K. TRUONG, "A 2D dynamic stall model based on a Hopf bifurcation", 19th European Rotorcraft Forum, Cernobbio, September 14-16, 1993.

[10] J.G. Leishman, T.S. BedDoEs, "A generalized model for unsteady airfoil behaviour and dynamic stall using the indicial method", 42nd Annual Forum of the American Helicopter Society, Washington D.C., June 1986.

[11] B. MichÉA, A. DESOPPER, M. COSTES, "Aerodynamic rotor loads prediction method with free wake for low speed descent flights", 18th European Rotorcraft Forum, Avignon, September 15-18, 1992.

[12] M. Costes, H.E. JoNEs, "Computation of transonic potential flow on helicopter rotor blades", 13th European Rotorcraft Forum, Arles, September 8-11, 1987.

[13] J.C. Boniface, "Calcul d'écoulements compressibles autour de rotors d'hélicoptères en vol stationnaire ou en vol d'avancement par résolution des équations d'Euler". Thèse de Doctorat, ENSAM, Décembre 1995.

[14] P. Beaumier, M. Costes, R. Gavériaux, "Comparison between FP3D full potential calculations and S1 Modane wind tunnel test results on advanced fully instrumented rotors", 19th European Rotorcraft Forum. Cernobbio, September 14-16, 1993.

[15] D. Petor, M. Allongue, "Aéroélasticité des rotors d'hélicoptères. Synthèse des travaux et perspectives", Revue Française de Mécanique, no 1995-1, pp. 57-66.

[16] G. Arnaud, P. Beaumier, "Validation of R85/METAR on the Puma RAE flight tests", 18th European Rotorcraft Forum, Avignon, September 15-18, 1992.

[17] P. BEAUMIER, "A coupling procedure between a rotor dynamics code and a 3D unsteady full potential code", AHS Aeromechanics Specialists Conference, San Francisco, January 19-21, 1994. 